Proceedings of the International Cenference on Symxhetic Metals

- To be published in Syntheric Merals

Gastein. Austria

- July 15-21, 2000

ANL/CHMICP-102622

The submitted manuscript has been authored

by a contractor of the U.S. Government

under contract No. W-31-109ENG-38.

Accordingly, the U.S. Government retains a nonexclusive, royalty-free license to publish

or reproduce the published form of this contribution, or allow others to do so, for Contribution, or allow others

\title{
Interplane transport effects in layered organic conductors
}

\author{
J.A.Symington ${ }^{2,1}$, J.Singleton $^{a}$, N.Harrison ${ }^{b}$, N.Clayton ${ }^{c}$, J.Schlueter $^{d}$, \\ M.Kurmoo, P.Day ${ }^{\mathrm{e}}$ \\ a Department of Physics, University of Oxford, the Clarendon Laboratory, Parks Road, Oxford, OX1 $4 A W$, UK. \\ ' National High Magnetic Field Laboratory, LANL Los Alamos, New Mexico 87545, USA. \\ ${ }^{c} H . H$.Wills Physics Laboratory, Tyndall Avenue, Bristol, BS8 1TL, UK. \\ 'Argonne National Laboratory, 9700 south Class Avenue, Argonne, IL 60439, USA. \\ e The Royal Institution, 21 Albemarle Street, London, $W 1 X 4 B C, U K$.
}

\begin{abstract}
Detailed studies of the magnetic field orientation on magnetic quantum oscillations in two charge transfer salts of the molecule ET have been carried out. After all conventional mechanisms affecting quantum oscillations have been accounted for, we find that the amplitude of the oscillations has an underlying dependence $\exp (-\alpha \tan \theta)$, where $\theta$ is the angle between the normal to the highly-conducting layers and the magnetic field, and $\alpha$ is a constant.
\end{abstract}

Key words: Organic superconductor, Transport measurements, magnetotransport, Magnetic measurements

Whether layered organic metals possess true three dimensional (3D) Fermi surfaces, or whether the interlayer transport is incoherent, has excited considerable recent interest (see e.g. [1,2]). As the temperature is raised, the resistivity in the interlayer direction rapidly passes the size at which the mean-free-path is smaller than the layer separation. McKenzie and Moses have thus proposed that the Fermi surface of ET salts are not necessarily extended in the interlayer direction [1]. They show that phenomena such as angle-dependent magnetoresistance oscillations [3] do not necessarily imply Fermi surfaces that are extended in the interplane direction; "weakly incoherent interlayer transport", in which a quasiparticle tunnels coherently between adjacent layers, but is scattered before subsequent tunneling processes, can give rise to similar phenomena. This paper reports quantum oscillation experiments carried out to detect any change in quantum oscillation behaviour at different field orientations. We find an anomalous angle dependence of the quantum oscillation amplitude which was observed in two very different ET charge-transfer salts and we

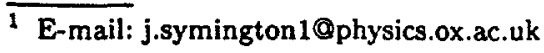

tentatively interpret this in terms of interplane tunnelling effects.

The samples studied were single crystals of $\alpha$-(ET) ${ }_{2} \mathrm{NH}_{4} \mathrm{Hg}(\mathrm{SCN})_{4}$ and $\beta^{\prime \prime}$ (ET) ${ }_{2} \mathrm{SF}_{5} \mathrm{CH}_{2} \mathrm{CF}_{2} \mathrm{SO}_{3}$, of approximate size $1 \times 1 \times$ $0.5 \mathrm{~mm}^{3}$. Both materials possess Fermi surfaces consisting of a quasi-two-dimensional (Q2D) pocket plus a pair of Q1D sheets [5]. De Haas-van Alphen oscillations were measured using standard field-modulation methods [5]. Temperatures down to $20 \mathrm{mK}$ were provided by a dilution refrigerator, and magnetic fields were generated by a $13.5 \mathrm{~T}$ superconductive solenoid. Shubnikov-de Haas oscillations were studied at fields of up to $33 \mathrm{~T}$ in a Bitter magnet at NHMFL, Tallahassee, using lowfrequency $\mathrm{AC}$ techniques described elsewhere [6]. In this configuration, the measured resistance is proportional to $\rho_{z z}[6]$. The samples were mounted in a cryostat which provided temperatures between $1.35 \mathrm{~K}$ and $4.2 \mathrm{~K}$, and allowed the sample to be rotated around two mutually perpendicular axes [6]. The amplitude $A$ of magnetic quantum oscillations of frequency $F$ caused by a Q2D Fermi surface should be described by the following variant of the Lifshitz-Kosevich formula [8]: 
Table 1

Experimentally determined values of $m^{*}, g^{*}$ and $T_{\mathrm{D}}$.

\begin{tabular}{|c|c|c|c|c|}
\hline Material & Expt. & $m^{*}\left(m_{0}\right)$ & $g^{*}$ & $T_{\mathrm{D}}(\mathrm{K})$ \\
\hline$\alpha-(\mathrm{ET})_{2}\left(\mathrm{NH}_{4}\right) \mathrm{Hg}(\mathrm{SCN})_{4}$ & $\mathrm{SdH}$ & 2.7 & 2.25 & 2.0 \\
$\alpha-(\mathrm{ET})_{2}\left(\mathrm{NH}_{4}\right) \mathrm{Hg}(\mathrm{SCN})_{4}$ & $\mathrm{dHVA}$ & 2.7 & 2.25 & 1.0 \\
$\beta^{\prime \prime}-(\mathrm{ET})_{2} \mathrm{SF}_{5} \mathrm{CH}_{2} \mathrm{CF}_{2} \mathrm{SO}_{3}$ & $\mathrm{SdH}$ & 2.0 & 2.05 & 2.0 \\
\hline
\end{tabular}

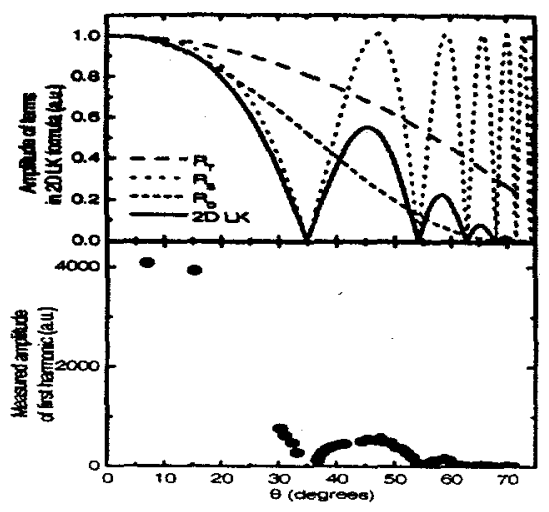

Fig. 1. Upper Figure: predicted amplitude of quantum oscillations in $\alpha$-(BEDT-TTF $)_{2} \mathrm{NH}_{4}(\mathrm{SCN})_{4}$ versus tilt angle $\theta$, obtained using Eqn. 1 and the parameters in Table 1. The dashed and dotted lines show the angle dependences of $R_{T}$, $R_{\mathrm{D}}$ and $R_{S}$; the solid line is Eqn. 1. The modulus of amplitude is shown. Lower Figure: measured amplitude of Shubnikov-de Haas oscillations in $\alpha$-(BEDT-TTF $)_{2} \mathrm{NH}_{4}(\mathrm{SCN})_{4}$ versus tilt angle $\theta$.

$$
A \propto\left(\frac{F}{B_{\mathrm{eff}}}\right)^{2} \cos \left(2 \pi\left[\left(\frac{F}{B_{\mathrm{eff}}}\right)-\frac{1}{2}\right]\right) R_{\mathrm{T}} R_{\mathrm{D}} R_{\mathrm{S}}
$$

where $\mathrm{B}_{\text {eff }}=B \cos \theta$. Here $R_{\mathrm{T}}, R_{\mathrm{D}}$ and $R_{\mathrm{S}}$ are damping terms due to finite temperature, finite scattering rate and electron spin, respectively [5].

Shubnikov-de Haas and de Haas-van Alphen oscillations obtained for each angle were Fourier transformed. Values of $m^{*}, T_{\mathrm{D}}$ and $g^{*}$ were found using conventional methods at $\theta=0^{\circ}[5]$. These values, summarised in Table 1 are in good agreement with previous studies [5].

The parameters from Table 1 are then used in Eqn. 1 to predict the angle-dependent amplitude of the quantum oscillations (Fig. 1; top section) which may be compared with the experimental angle dependence (Fig. 1; bottom section). It is obvious from Fig. 1 that the experimental amplitudes fall off more rapidly with increasing angle than do the theoretical predictions.

Fig. 2 shows the experimental quantum oscillation amplitudes divided by Eqn. 1 plotted against $\tan \theta$ using a logarithmic vertical scale. There is some scatter of experimental points caused by the difficulty of accurate normalisation close to the spin zeros. However, it is plain that the data for both materials and for the two different techniques map roughly onto straight lines, i.e., the experimental angle dependence of the amplitude $A$ of

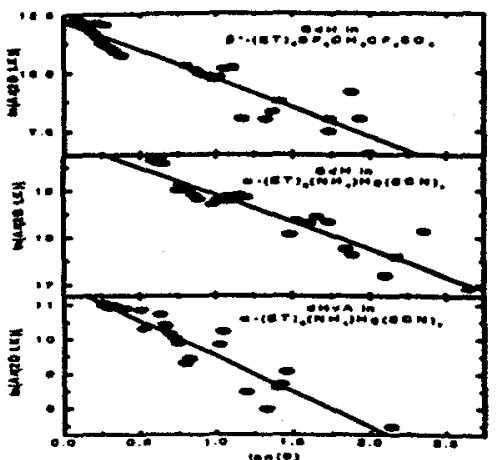

Fig. 2. Natural logarithm of the ratio of experimental quantum oscillation amplitude to the prediction of Eqn. 1 versus $\tan \theta$. The lines are straight-line fits to the data.

magnetic quantum oscillations includes a term proportional to $\exp (-\alpha \tan \theta)$, where $\alpha$ is a constant.

The cause of this extra damping term is unclear, but one possibility is an interlayer, inter-Landau level coupling caused by the in-plane component of the field [9]. This could lead to an apparent broadening of the Landau levels scaling as $\tan \theta$. Simulations are being carried out to investigate this possibility.

In summary, we have demonstrated a new damping term $\exp (-\alpha \tan \theta)$ in the angle dependence of the amplitude of the quantum oscillations of two quite different ET salts. The effect occurs in both magnetisation and magnetoresistance, suggesting that it might be a very general phenomenon in Q2D organic conductors.

This work is supported by EPSRC (UK). NHMFL is supported by the Department of Energy, the National Science Foundation and the State of Florida.

\section{References}

[1] P. Moses and R.H. McKenzie, Phys. Rev. B 60, 7998 (1999); R.H. McKenzie and Perez Moses, Phys. Rev. B 60, R11241 (1999).

[2] D. Yoshioka, J.Phys. Soc. Jpn 64, 3168 (1995).

[3] S.J. Blundell, J. Singleton, J. Phys. I. France, 6, 1837, (1996).

[4] K. Yamaji, J. Phys. Soc. Jpn. 58, 1520 (1989).

[5] J. Singleton, Reports on Progress is Physics, 63, 675 (2000).

[6] M.-S. Nam et al. J. Phys.: Condens. Matter, 11, L477 (1999).

[7] A.A. House et al., J. Phys.: Condens. Matter 8, 8829 (1996).

[8] N. Harrison et al., Phys. Rev. B. 54), 9977 (1996) and references therein.

[9] J. Chalker et al., unpublished. 


\section{DISCLAIMER}

This report was prepared as an account of work sponsored by an agency of the United States Government. Neither the United States Government nor any agency thereof, nor any of their employees, make any warranty, express or implied, or assumes any legal liability or responsibility for the accuracy, completeness, or usefulness of any information, apparatus, product, or process disclosed, or represents that its use would not infringe privately owned rights. Reference herein to any specific commercial product, process, or service by trade name, trademark, manufacturer, or otherwise does not necessarily constitute or imply its endorsement, recommendation, or favoring by the United States Government or any agency thereof. The views and opinions of authors expressed herein do not necessarily state or reflect those of the United States Government or any agency thereof. 


\section{DISCLAIMER}

Portions of this document may be illegible in electronic image products. Images are produced from the best available original document. 